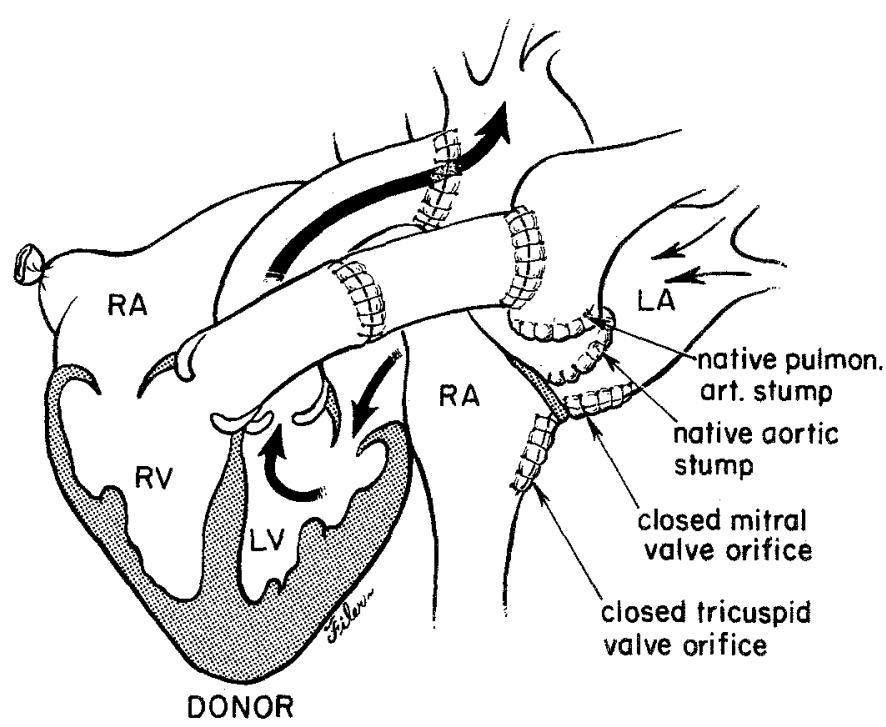

Fig. 2. Native cardiectomy at atrioventricular junction with closure of native atrial, aortic, and pulmonary arterial stumps. Arrows indicate flow. $R A$, Right atrium; $L A$, left atrium; $R V$, right ventricle; $L V$, left ventricle.

had an infected aortic valve prosthesis of the native heart. That patient survived 8 years with this "hybrid" heart. This option is suitable for those patients who still have pulmonary hypertension and therefore require a functional native right ventricle.

Another option is to perform a native cardiectomy, as was done in this case. This option is feasible only when the transplanted heart is able to function without the assistance of the native right ventricle. Our patient had demonstrated that the transplanted heart was able to support the entire circulation for a period of 9 months while the native heart was in ventricular fibrillation. Native cardiectomy is a more extensive operation than closure of the aortic valve; however, the potential problem of thrombus formation and endocarditis in the native heart is eliminated.
We thank David C. K. Cooper, MD, PhD, and Dimitri Novitzky, MD, for their valuable advice.

\section{REFERENCES}

1. Desruennes M, Muneretto C, Gandjbakhch I, Kawaguchi A, Pavie A, Bors V, et al. Heterotopic heart transplantation: current status in 1988. J Heart Transplant 1989;8:479-85.

2. Hildebrandt $A$, Reichenspurner $H$, Gordon GD, Horak AR, Odell JA, Reichart B. Heterotopic heart transplantation: mid-term hemodynamic and echocardiographic analysis--the concern of arteriovenous-valve incompetence. $J$ Heart Transplant 1990;9:675-82.

3. Losman JG, Curcio CA, Barnard CN. Normal cardiac function with a hybrid heart. Ann Thorac Surg 1978;26:177-80.

\title{
CLINICAL ENDOVASCULAR PLACEMENT OF BRANCHED GRAFT FOR TYPE B AORTIC DISSECTION
}

Kanji Inoue, MD, ${ }^{a}$ Mitsuru Sato, ME, ${ }^{\mathrm{b}}$ Tomoyuki Iwase, MD, ${ }^{\mathrm{c}}$ Yuki Yoshida, MT, ${ }^{\mathrm{b}}$ Terumitsu Tanaka, MD, Shunichi Tamaki, MD, ${ }^{\mathrm{c}}$ and Ario Yamazato, MD, Kyoto, Japan

The concept of a catheter-based strategy for managing aortic dissections and aneurysms continues to be exciting and promising. Most type $\mathrm{B}$ aortic dissections have initial

From the Departments of Cardiovascular Surgery, ${ }^{a}$ Clinical Laboratory, ${ }^{b}$ and Cardiology, ${ }^{\mathrm{c}}$ Takeda Hospital, Kyoto, Japan.

Received for publication Jan. 18, 1996; accepted for publication Feb. 15, 1996.

J Thorac Cardiovasc Surg 1996;112:1111-3

Copyright (c) 1996 by Mosby-Year Book, Inc.

$0022-5223 / 96 \$ 5.00+0 \quad \mathbf{1 2 / 5 4 / 7 2 8 9 7}$ intimal tears just beyond the left subclavian artery, and a straight graft with a side branch would be necessary to obliterate such a tear. We report the successful placement of a branched graft in a patient with type B aortic dissection.

A 51-year-old woman who was believed to have acute aortic dissection was referred to our hospital on May 9 , 1995. The patient's condition was managed medically because the false lumen of the descending thoracic aorta was found to be completely thrombosed by transesophageal echocardiography and contrast computed tomography. Transesophageal echocardiography during admission demonstrated that the false lumen gradually disappeared. 

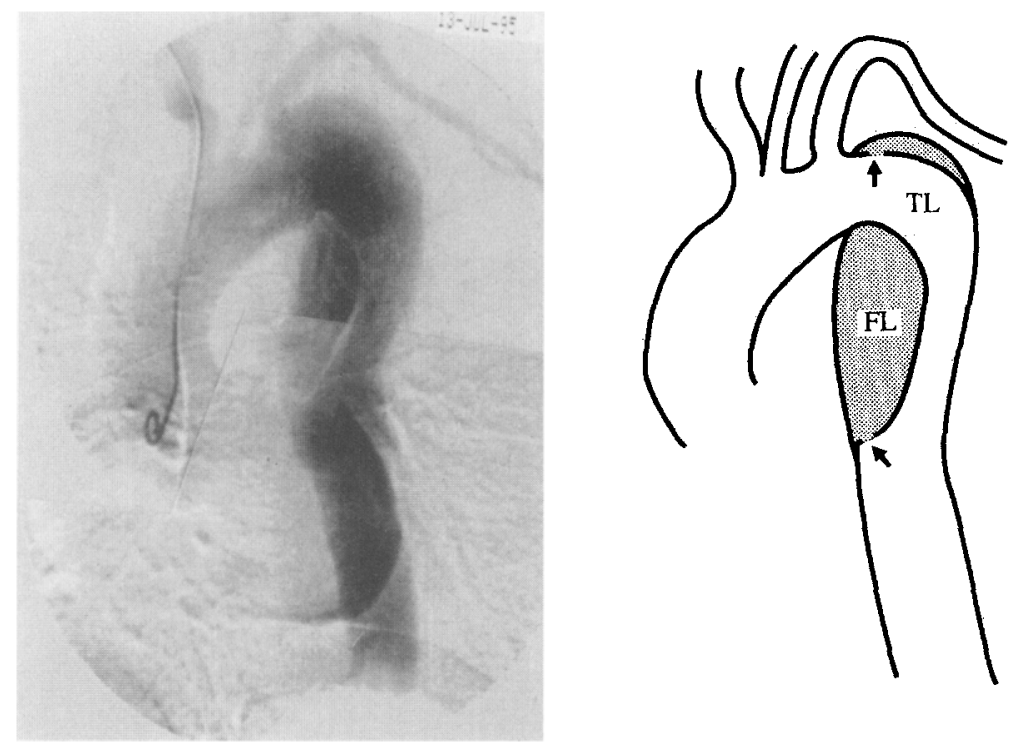

Fig. 1. Before endovascular graft placement, aortography shows large entry site just beyond left subclavian artery (upper arrow) and reentry site in descending thoracic aorta (lower arrow). TL, True lumen; $F L$, false lumen.

The patient recovered favorably with medical management and was discharged from our hospital. She was readmitted, however, after reporting severe back pain on June 22, 1995. Transesophageal echocardiography revealed a large entry just beyond the left subclavian artery with reentry in the descending thoracic aorta, approximately $5 \mathrm{~cm}$ below the entry site. The false lumen was patent without thrombus formation. These findings were confirmed by aortography (Fig: 1 ) atid contrast computed tomography. After a full description regarding potential complications had been given to the patient and the possibility of emergency operation had been explained to her, the patient gave informed consent for the experimental alternative of endovascular graft placement.

A new graft and a delivery system were designed and developed by Inoue and colleagues. ${ }^{1,2}$ The graft was constructed from a Dacron polyester fabric cylinder, and the surface was supported by multiple rings of extraflexible nickel titanium wire. The rings at both ends of the graft were covered by loosely spun Dacron polyester filaments so that the graft attached closely to the aortic wall. Two thorns made of wire, 3 to $5 \mathrm{~mm}$ in length, were attached at each middle ring. The thorns ensured the graft fixation by hooking into the aortic wall. The size of the tapered straight graft was $34 \mathrm{~mm}$ in diameter at the proximal end, $28 \mathrm{~mm}$ in diameter at the distal end, and $210 \mathrm{~mm}$ in length. The side-branch graft was $8 \mathrm{~mm}$ in diameter and $30 \mathrm{~mm}$ in length. The diameter, length, and taper were based on measurements obtained from transesophageal echocardiography, computed tomography, and magnetic resonance imaging.

After initial aortography, the right femoral artery was surgically isolated and a transverse arteriotomy was performed with the patient under local anesthesia. A $22 \mathrm{~F}$ sheath was introduced into the descending thoracic aorta over a 0.038 -inch guidewire previously placed through the femoral arteriotomy under fluoroscopic guidance. The branched graft was evenly folded with loops of thread and a nickel titanium wire. The branched graft and its carrying system were introduced within the sheath and advanced to the descending thoracic aorta under fluoroscopic monitoring. During the fixing of the sheath in the descending thoracic aorta only the graft was advanced to the aortic arch. After the branched graft was positioned at the predetermined target point, the free end of a detachable wire attached to the side-branch graft was caught and pulled back by a gooseneck snare wire, which was inserted percutaneously through the left brachial artery. After the side-branch graft had been placed in the left subclavian artery, the compactly folded branched graft was deployed by removal of the nickel titanium wire, allowing rapid expansion of the branched graft by its own flexibility. Finally, the branched graft was released from the carrying system and pressed against the aortic wall by balloon inflation. Aortography was repeated to confirm that the branched graft was properly positioned and that the entry and reentry sites were completely obliterated. The arteriotomy site was subsequently repaired.

The straight graft with a side-branch graft was successfully and uneventfully implanted. Immediately after the procedure, aortography showed good flow of contrast medium through the graft, with small leakage through the graft texture into the false lumen as a result of intraoperative heparinization. The patient did not require blood transfusion and was fully ambulatory after 3 days. One month after the procedure, aortography and contact computed tomography demonstrated that the false lumen had completely thrombosed, with no residual filling, and the flow through the graft was normal (Fig. 2). Although transesophageal Doppler echocardiography detected trivial patent proximal tracts communicating with the false lumen, the false lumen had almost completely thrombosed.

Various types of grafts and stents have been developed 

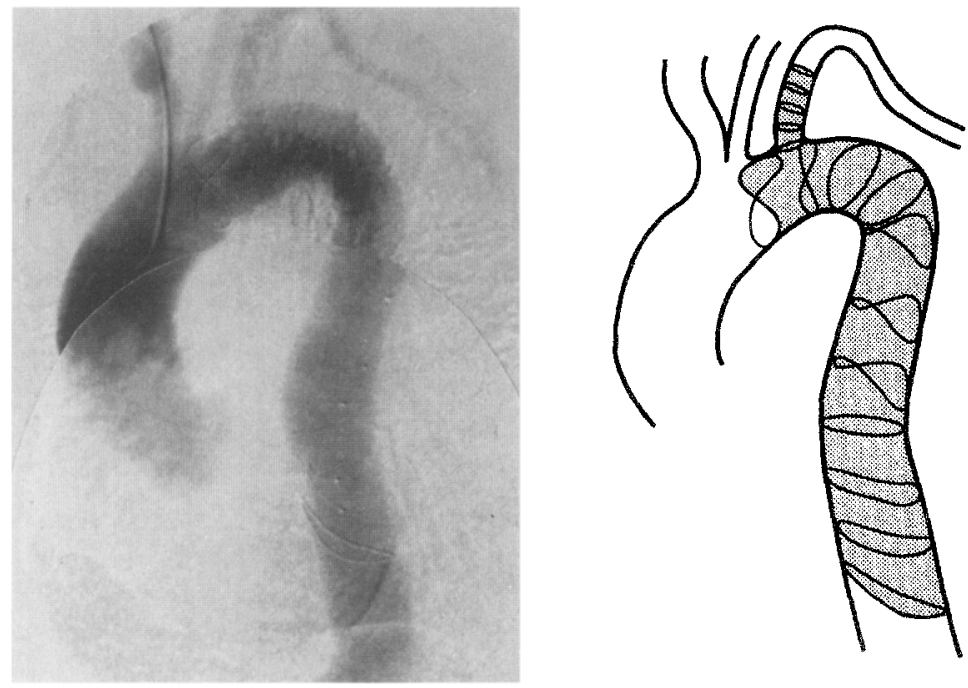

Fig. 2. One month after graft placement, aortography shows good flow of contrast medium through graft, with no leakage into false lumen.

since 1969, when Dotter ${ }^{3}$ initially inserted stainless steel coils as a vascular stent in canine popliteal arteries. Clinical endovascular placement of straight stent grafts for the treatment of aortic dissection has already been reported, ${ }^{4}$ but placement of these stent grafts was limited to sites that did not include the origin of the major aortic arterial branches. With the branched graft, we succeeded in endovascular repair of type B dissection with intimal tear just beyond the left subclavian artery. If long-term results are favorable, endovascular repair of larger dissections in patients with type $B$ dissection would be possible. Careful long-term evaluation and further improvement in devices and techniques will, however, be necessary for extensive clinical use.

\section{REFERENCES}

1. Inoue $\mathrm{K}$, Htay T, Kida M, Fujiwara $\mathrm{H}$. Percutaneous implantation of aortic endovascular graft for created aneurysm: animal experiment [abstract]. Circulation 1991;84(Suppl):n421.

2. Inoue $\mathrm{K}$, Htay $\mathrm{T}$. Long-term follow-up of percutaneously placed aortic endovascular graft: animal experiment [abstract]. Circulation 1992;86(Suppl 1):I636.

3. Dotter CT. Transluminally-placed coilspring endoarterial tube grafts: long-term patency in canine popliteal artery. Invest Radiol 1969;4:329-32.

4. Dake MD, Miller DC, Semba CP, Mitchell RS, Walker PJ, Liddell RP. Transluminal placement of endovascular stentgrafts for the treatment of descending thoracic aortic aneurysms. N Engl J Med 1994;331:1729-34.

\section{AN ADJUSTABLE, PERCUTANEOUSLY PLACED HELICAL COIL: CONTROL OF LEFT VENTRICULAR FILLING PRESSURE DURING CARDIOPULMONARY BYPASS IN SEVERE LEFT VENTRICULAR FAILURE}

Theodor Kolobow, MD, Jeffrey Wang, Matteo Giacomini, MD, and Chiara Reali-Forster, MD, Bethesda, Md.

We previously reported that closed-chest total cardiopulmonary bypass through peripheral cannulation can be performed in sheep with induced ventricular fibrillation

From the Pulmonary-Critical Care Medicine Branch, National Heart, Lung, and Blood Institute, National Institutes of Health, Bethesda, Md.

Received for publication Nov. 1, 1995; accepted for publication Feb. 26, 1996.

J Thorac Cardiovase Surg 1996;112:1113-5

Copyright (C 1996 by Mosby-Year Book, Inc.

$0022-5223 / 96 \$ 5.00+0 \quad \mathbf{1 2 / 5 4 / 7 3 1 0 2}$ for at least 3 days, with immediate recovery of normal cardiac and pulmonary function after defibrillation. ${ }^{1}$ Key to the success of this procedure was the development of a percutaneously placed, fixed-size helical coil that is advanced to rest within the lumen of the pulmonary artery valves and the tricuspid valves to keep them open. This allows the retrograde flow of blood from the bronchial venous system and the thebesian venous flow and from the left side of the heart across the lungs (with the heart in ventricular fibrillation), decompressing the left side of the heart. In this article we describe the design and development of a flow-directed, catheter-mounted helical coil system that has an adjustable diameter and is therefore 